\title{
Common skin disorders in the elderly
}

Omar $\boldsymbol{M} \boldsymbol{H}, \mathrm{MBChB}(\mathrm{UCT})$, Dip. Occ. Health (U.S.)

Senior Consultant, The Albertina and Walter Sisulu Institute of Ageing in Africa, Faculty of Health Sciences, University of Cape Town

Correspondence: Dr Mohamed Omar, E-mail: homar@uctgsh1uct.ac.za

\section{Abstract}

Ageing causes a decline in the function of human skin, while factors such as medical conditions, drugs and environmental irritants add to the compromised skin and predispose it to certain conditions. Superimposed on the changes of physiological ageing are changes characterised by chronic sun exposure. Skin neoplasia, whether benign, premalignant or malignant, is more common in the elderly. It is important to identify benign conditions, as it is crucial that lesions with a malignant potential be recognised so that timeous treatment can prevent serious malignancies. Ultraviolet radiation is the major aetiologic factor for the development of skin cancer. Pruritic conditions result from a combination of a declining barrier function and the effects of environmental irritants. Pruritus due to scabies is common in institutionalised older persons. Infective conditions as a result of a combination of altered immunity, predisposing medical conditions (e.g. diabetes) and a variety of drugs used to treat these conditions may affect immune function and homeostasis. Regular scrutiny of the skin will ensure early identification of problems and implementation of a good skin care plan can compensate for failing physiologic function.

SA Fam Pract 2006;48(5): 29-34)

\section{INTRODUCTION}

Population ageing and a drive to maintain a youthful appearance have spurred research on the physiological processes of skin ageing. An important outcome of these efforts has been greater insight into skin cancer.

Tremendous strides have also been made in the last decade in understanding the molecular basis of ageing. The free radical theory states that ageing results from an accumulation of cellular damage caused by excess reactive oxygen species generated by oxidative metabolism. ${ }^{1}$ The role of tumour suppressor genes, particularly the p53 pathway, has received increasing attention. As cell division can lead to mutations, and hence cancer, organisms have developed tumour suppressor mechanisms. These suppressor genes act on cells, causing them to die (apoptosis) or to arrest proliferation (senescence) permanently. However, this process acts as a double-edged sword, restraining the development of cancer by eliminating or preventing the growth of potential cancer cells, while at the same time having a pro-ageing effect through the accumulation of senescent cells. ${ }^{2}$ Senescent cells can alter the local tissue milieu, resulting in the hyperproliferative diseases of ageing, including cancer. ${ }^{3}$

An accumulation of senescent cells also results in a decline in several functions of the human skin, including barrier efficacy, sensory perception, wound healing, immune responsiveness and DNA repair. The impact of these changes varies from cosmetic to life-threatening disorders. Though varying in severity, all may adversely affect an older person's health and quality of life.

A distinction must be made between intrinsic ageing attributable to the passage of time, and photoageing, which is characterised by changes due to chronic sun exposure superimposed on intrinsic ageing. ${ }^{4}$ The latter features are most noticeable in fair-skinned individuals.
Dryness, purpura, actinic keratosis, telangiectasia, wrinkling, coarseness and irregular pigmentation are some of the indicators of photoaged skin. As the black population increasingly embraces a Western lifestyle, the consequences of sunburn and leisure sun exposure in this population are still unknown.

\section{Figure 1: Photoageing}

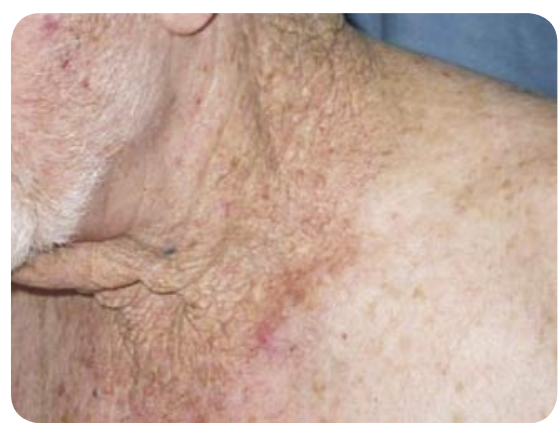

\section{NEOPLASIA}

With increasing age, the body's ability to repair damage is decreased, resulting in greater risk of neoplastic growth. Benign, premalignant and malignant neo-plasia are discussed briefly. 


\section{Benign neoplasia}

Benign proliferative lesions are common in the elderly, often increasing in number and size with advancing age. They should be distinguished from malignant or premalignant lesions.

- Skin tags, or fibroepithelial polyps, are skin-coloured to pigmented pedunculated papules, which often occur on the eyelids, axillae and neck.

Figure 2: Skin tags

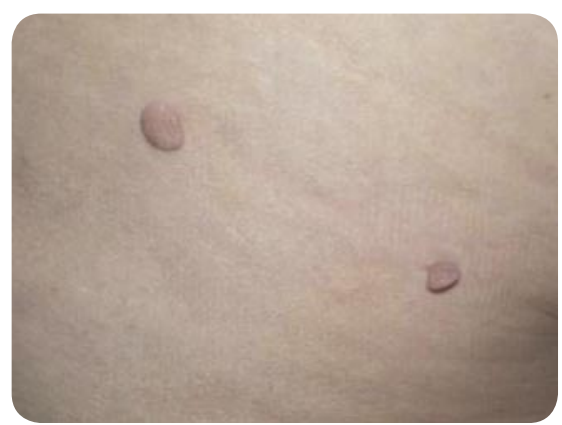

- Seborrhoeic keratosis manifests as brown macules, papules or plaques and may range in colour from white to black. They are called seborrhoeic warts because of the verrucous or "stuck on" appearance. The condition is common on the face, neck and trunk.

Figure 3: Seborrhoeic dermatitis

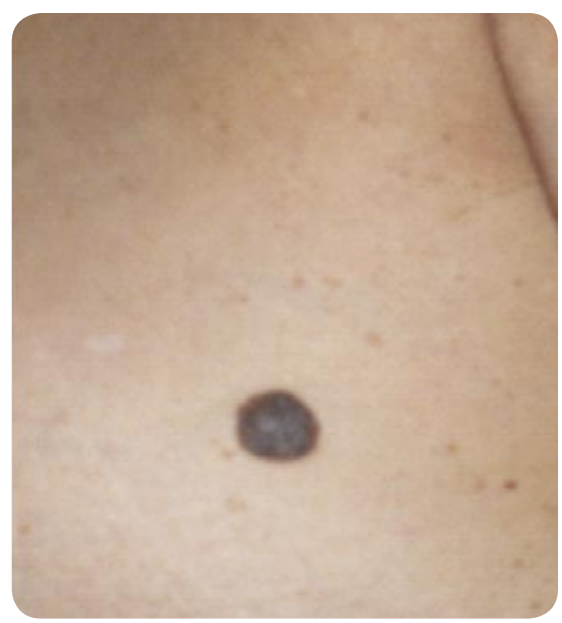

- Cherry angiomas (Campbell de Morgan spots) are dilated, congested capillaries and venules. Ruby red papules up to $6 \mathrm{~mm}$ in size, they are usually found on the trunk and proximal part of limbs.
- Solar lentigines are tan to brown macules 2-4 mm in diameter that gradually increase in size and number and may coalesce into larger patches. The common sites are skin areas exposed to sunlight.

Treatment of the above conditions is done mainly for cosmetic reasons. They can be treated by cryotherapy, cautery or, as a last resort, by simple surgical excision. Should there be any doubt as to the diagnosis, the excised skin specimen should be sent for histological examination.

\section{Premalignant neoplasia}

Premalignant neoplasia are lesions with the potential for progressing to malignancy. Early recognition and timeous treatment may prevent serious skin malignancies.

- Actinic keratosis is a common precursor neoplasia of sundamaged skin and may develop into squamous cell carcinoma. As many of these lesions regress spontaneously and only very few progress to malignancy, their management and premalignant potential is controversial. ${ }^{5}$ Lesions appear as rough, red, scaly papules. Early lesions are more easily palpated than seen; a cutaneous horn may form only rarely. Treatment involves cryotherapy, or topical imiquimod or 5fluorouracil. ${ }^{6}$ Photodynamic therapy has also been used successfully. Recurrence of the lesion at the same site requires surgical excision and histological examination to exclude an early malignancy.

Figure 4: Actinic keratosis

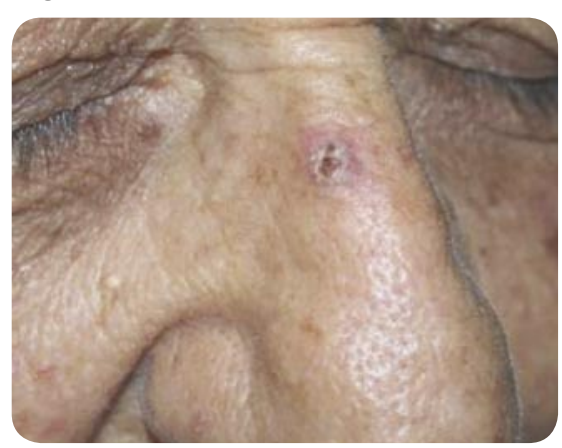

- Bowen's disease is a full thickness intra-epidermal carcinoma that presents as a well circumscribed red, scaly solitary plaque. The plaques occur on sun-exposed areas and must be differentiated from nummular eczema and psoriasis. Any scaly lesion in sundamaged skin unresponsive to topical therapy should be biopsied. Topical imiquimod or 5-fluorouracil, or surgery, is used to treat lesions.

- Lentigo maligna is an "in situ" melanoma of chronically sundamaged skin. Common sites are the face, especially the nose and cheeks. Lentigo maligna appears as a slowly enlarging macule with irregular borders and variegate pigmentation. The condition must be differentiated from solar lentigo, which are smaller and have homogeneous colour and regular borders. Dermatoscopy, in expert hands, can be a useful tool to help distinguish between benign and malignant lesions. Treatment options for lentigo maligna include surgical excision with a narrow margin, laser surgery and cryotherapy. Close observation may be the chosen option in selected cases, because lentigo maligna could exist for many years before an invasive melanoma appears.

Figure 5: Lentigo maligna

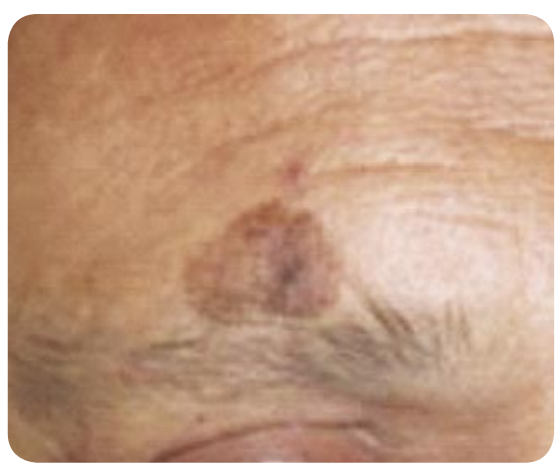

\section{Malignant neoplasia}

The major aetiologic factor for skin cancer is ultraviolet radiation. Cumulative sun exposure and a high frequency of intense intermittent exposures also contribute to certain cancers. ${ }^{7}$ For this reason, skin cancer is often found against 
a background of sun damage. Immunosuppression, carcinogenic agents (betel nut, tobacco) and viruses (human papilloma virus) also contribute to the pathogenesis. Types of non-melanoma and malignant melanoma skin cancers are:

\section{- Squamous cell carcinoma,} non-melanoma, involves the extension of neoplastic cells into the dermis. The condition arises as a skin-coloured to red papule, plaque or nodule on a sun-exposed area. It may be friable and can bleed with slight trauma.

Figure 6: Squamous cell carcinoma

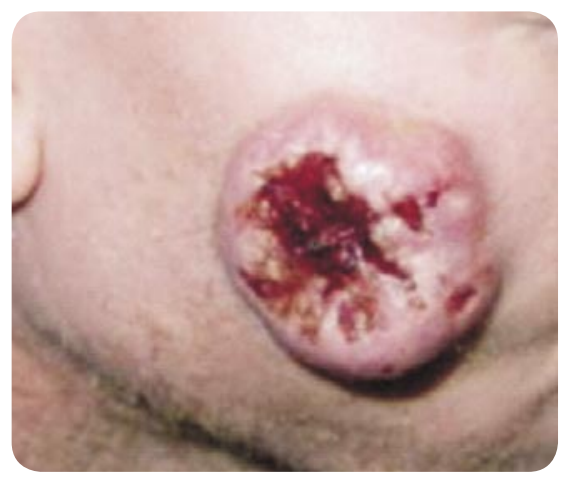

- Basal cell carcinoma, also non-melanoma, is a translucent (pearly) skin-toned to pink papule or plaque with overlying telagiectasia. It may ulcerate. The head and neck are the most common sites of occurrence, but it can occur on any exposed skin. Treatment options of non-melanoma skin cancer include curettage and cautery, or excision and radiation. In certain cases, cryosurgery, $\mathrm{CO}_{2}$ laser, photodynamic therapy and topical imiquimod or 5-fluorouracil may be used. ${ }^{8}$ Although these conditions may be managed by the general practitioner, it is essential that the excised or curetted specimens be sent for histology. Referral for specialist removal is suggested for lesions on a cosmeticallysensitive site such as the face, for recurrences or for rapidly growing lesions. Patients with basal cell carcinoma are at high risk of developing recurrences and need to be cautioned about limiting sun exposure and the need for longterm follow up. ${ }^{9}$

Figure 7: Basal cell carcinoma

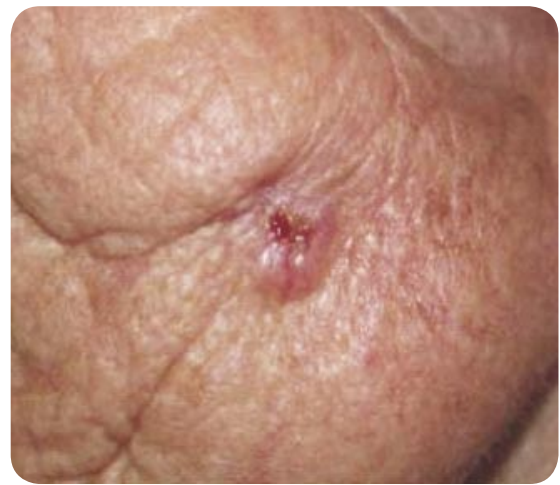

\section{- Malignant melanoma is}

increasing in incidence worldwide. In addition to exposure to sunlight, other risk factors include preexisting pigmented lesions and a family history of malignant melanoma. Suspicion of malignant melanoma in any pigmented lesion follows the "ABCD" rule:

- Asymmetry

- Border irregularity

- Colour variation or dark black colour

- Diameter greater than $6 \mathrm{~mm}$

Hence, any change in the shape, colour or size of a pigmented lesion should arouse suspicion of cancer. The ugly duckling sign refers to a melanotic lesion that is atypical beyond the context of surrounding naevi. ${ }^{10}$ This lesion should be regarded with suspicion regardless of specific findings. Malignant melanoma can occur anywhere on the skin and is often seen on the back, head and neck (males), lower extremities (females), and the palms and soles (blacks). Early detection is vital, as the prognosis depends on the depth of penetration of the lesion. A lesion that is clinically suspicious for melanoma should ideally undergo an excisional biopsy with narrow margins (2 $\mathrm{mm})$. An incisional biopsy should be reserved for cases in which the tumour is too large to be excised, or when it is impractical to perform an excision. Once melanoma is confirmed, referral to a specialist centre for further management is advised.

\section{PRURITIC CONDITIONS}

Pruritus is a very common complaint among the elderly. The natural attrition of the adnexal glands that moisturise the skin and its decreased barrier function, together with the effects of irritants (soaps), the environment and sun exposure combine to produce dry, irritable skin. All itchy conditions may lead to excoriations and secondary bacterial infection. Pruritic conditions include:

- Asteatosis. Dry skin is almost universal in the elderly, and frequent bathing, low humidity, rough clothing and poor nutrition aggravate the condition. Asteatosis is characterised by a dull, fine scale with a crazy paving appearance. With scratching and irritation it becomes red, weepy and crusted (asteatotic eczema). Although it may start on the shins, the involvement of the rest of the body may occur.

The avoidance of exacerbating factors and the liberal use of emollients are the mainstay of management. Short courses of topical steroids may be necessary to control inflammatory flares.

- Pruritus. Besides asteatosis, many other cutaneous diseases can result in marked itching. In the absence of skin lesions, psychological factors or systemic diseases should be considered. Iron deficiency anaemia, thyroid dysfunction, renal failure, cholestasis, diabetes and myeloproliferative disorders are the systemic diseases that need to be considered. Adverse drug reactions can also manifest predominantly or exclusively as pruritus. The underlying disorder needs to be managed to control the itch.

- Scabies. Old-age homes provide a fertile ground for the rapid spread of the infestation. Scabies 
is often misdiagnosed because of atypical presentations and confusion with asteatosis. The diagnostic mite burrow is found on the wrists, web spaces, posterior axillae, areolae, periumbilical skin and penile shaft. The inflammatory, itchy skin lesions are due to a hypersensitivity reaction and can persist for a few weeks - even after effective treatment of the infestation.

Treatment is difficult because of re-infestation and poor compliance. Benzyl benzoate applied to the body for 12 hours is recommended. The treatment should be repeated a week later and contacts must also be treated. Another option is the use of pyrethroid sprays which are scabicidal. Topical steroids are helpful in alleviating the itch.

\section{Figure 8: Scabies}

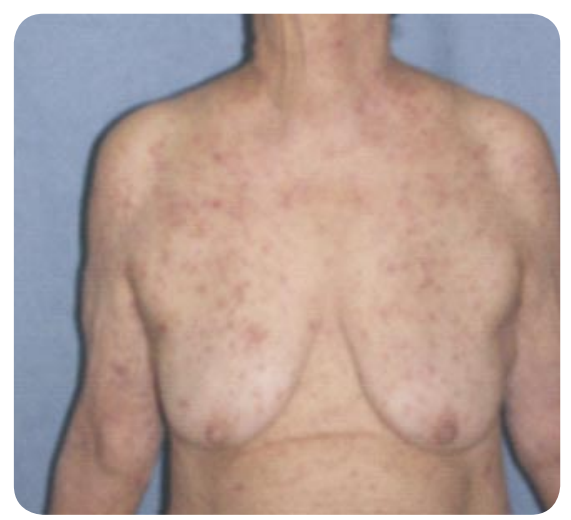

- Intertrigo is a mechanical, frictional problem in the flexures, with frequent secondary infection by Candida. It is especially common in diabetics. The inflammation is treated with topical steroids and maceration can be minimised with moisture-absorbing powders. Secondary infection may be treated with topical azoles or nystatin.

\section{Blistering conditions}

Blistering diseases in older patients may be life threatening. The clinical differential diagnosis may be difficult, but can be clarified by considering factors such as the age of the patient at onset, the pattern and distribution of the blisters, the presence of the Nikolsky sign and the drug history.

- Bullous pemphigoid is an autoimmune disorder characterised by antibodies to the dermoepidermal junction. It is the most common of the auto-antibodymediated blistering disorders in the elderly. It may present initially as urticarial papules or plaques, which then develop into large tense blisters. Lesions occur predominantly on the trunk and limbs, with the flexures being a common site. Healing with scarring occurs rarely, more often resolving with hyper- or hypopigmentation. The course is one of remissions and exacerbations. Diagnosis is confirmed by biopsy and immunofluorescence studies. The mainstay of treatment is systemic prednisone. At times, azathioprine may be used as a steroid-sparing agent. Localised bullous pemphigoid and mild intermittent flares can be managed with potent topical steroids. Some studies suggest that tetracyclines, nicotinamide and dapsone can be used as alternatives to the above treatment.

Figure 9: Bullous pemphigoid

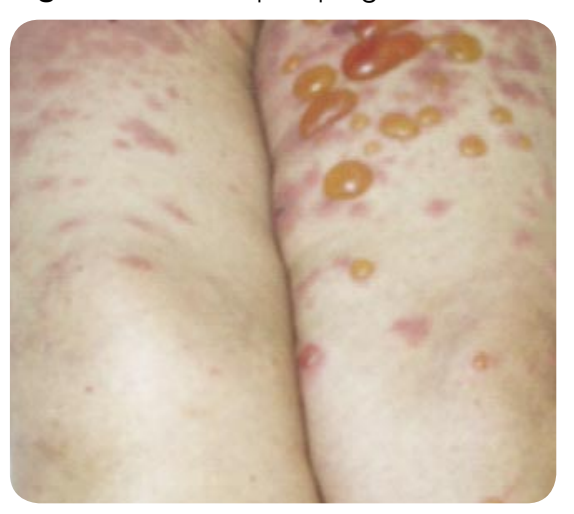

- Herpes Zoster entails a reactivation of the chicken pox virus, which lies dormant in the dorsal root ganglion. It begins with pain and burning, followed by the appearance of grouped vesicles on an erythematous base. The vesicles follow a dermatomal distribution. Eye involvement may result in serious conjunctivitis, iritis or uveitis. Post-herpetic neuralgia occurs predominantly in the elderly and is often severely debilitating. Calamine lotion may be used for intact vesicles, but silver sulphadiazine may be better employed for eroded lesions. Systemic acyclovir or its analogues, if started early, can shorten the course of the disease and prevent post-herpetic neuralgia. Treatment options for post-herpetic neuralgia include topical capsaicin, amitriptyline and carbamazepine.

Figure 10: Herpes Zoster

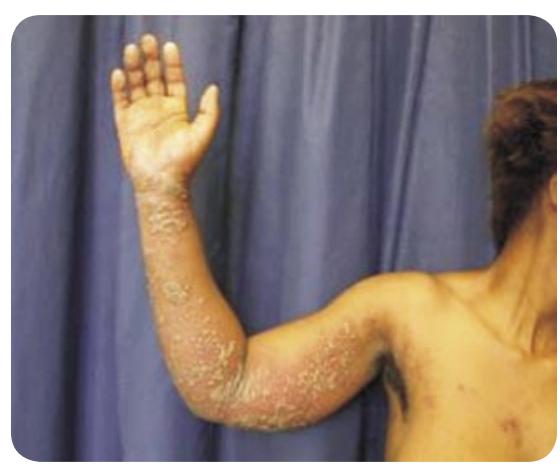

\section{ULCERATING CONDITIONS}

The development of ulcers and their failure to heal have a multifactorial basis in the elderly. Nutritional status, co-existing systemic disease and "skin failure" all play a role.

- Leg ulcers are most often secondary to venous insufficiency, arterial disease, neuropathy, or a combination of these factors. The three major types of leg ulcers are compared in Table I. General measures include good nutrition, treating any infection and using dressings that promote moistwound healing. Specific treatment requires attention to the underlying cause:

- Venous ulcers will require compression, elevation and exercise to relieve the oedema.

- The main goal of therapy in arterial ulcers is the re-establishment of an adequate arterial supply.

- The cause of most diabetic foot ulcers is repetitive trauma, and education and preventative measures are vital. 
Table I: Comparison of three major types of leg ulcers in elderly patients

\begin{tabular}{|c|c|c|c|}
\hline & Venous & Arterial & Neuropathic \\
\hline Symptoms & $\begin{array}{l}\text { Heaviness associated with } \\
\text { swelling }\end{array}$ & $\begin{array}{l}\text { Claudication } \\
\text { Pain }\end{array}$ & $\begin{array}{l}\text { Burning } \\
\text { Itching }\end{array}$ \\
\hline Location & Malleolar region & Pressure sites & Pressure sites \\
\hline Morphology & Irregular borders & $\begin{array}{l}\text { Necrotic base } \\
\text { "Punched out" }\end{array}$ & "Punched out" \\
\hline Surrounding skin & $\begin{array}{l}\text { Pigmentation (haemosiderin) } \\
\text { Lipodermatosclerosis } \\
\text { (firm, indurated, } \\
\text { woody) }\end{array}$ & $\begin{array}{c}\text { Shiny atrophic } \\
\text { skin with hair loss }\end{array}$ & Thick callus \\
\hline $\begin{array}{l}\text { Other clinical } \\
\text { findings }\end{array}$ & $\begin{array}{l}\text { Varicosities, oedema } \\
\text { +/- Stasis dermatitis } \\
\text { +/- Lymphoedema }\end{array}$ & $\begin{array}{l}\text { Weak/absent peripheral pulses } \\
\text { Prolonged capillary refilling time } \\
\text { Pallor on leg elevation }\end{array}$ & $\begin{array}{l}\text { Peripheral neuropathy with } \\
\text { decreased sensation }\end{array}$ \\
\hline
\end{tabular}

- Pressure ulcers usually develop on any part of the body where there is sustained pressure under the right circumstances and usually appear over bony prominences. The pressure ulcer has various manifestations, depending on the stage. There may be superficial or deep ulcers with eschar or gangrene formation. Treatment includes relieving pressure by regular turning and use of either static or dynamic pressurerelieving support surfaces. Infection must be managed and moist wound healing is the preferred treatment.

It is important to be certain that a presumed vascular ulcer is not actually a skin cancer. Indications that an ulcer is cancerous include ulcers that are heaped up, partially keratinised, and have a prominently raised border or simply fail to heal in a timely manner.

\section{INFECTIONS}

The diagnosis and management of infectious diseases of the skin are significant issues in the elderly. It is likely that underlying diseases, such as diabetes, may predispose to more frequent and severe infections.

- Tinea pedis is common and is usually exacerbated with age. Interdigital tinea pedis in elderly diabetics may ulcerate and predispose to cellulitis. The involved nails may act as a reservoir and more aggressive treatment is necessary to effect a cure. Oral azoles, terbinafine or long-term griseofulvin are recommended. Topical anti-fungal nail paints may also be of value. In the absence of onychomycosis, topical antifungals and moisture-absorbing powders may suffice.

\section{- Cellulitis and erysipelas}

present as acute problems that can be life threatening in the elderly. Predisposing factors include $T$. pedis, oedema, dry skin, diabetes and compromised circulation. Streptococcal infection requires either parenteral penicillin or ery-thromycin for at least 10 days. Staphylococci can be eradicated with flucloxacillin. Augmentin or clindamycin are equally effective in treating infections caused by both micro-organisms. For recurrent infections, it is advisable to look for a predisposing factor and to maintain long-term control with monthly parenteral long-acting penicillin.

Superficial infections require aggressive treatment, as they present a significant problem for elderly diabetic, immuno-compromised and vascular-incompetent persons. A low-grade infection allows other bacteria an excellent portal of entry. Proper foot and leg care are essential and may require referral to a podiatrist.

\section{CONCLUSIONS}

All skin lesions in the elderly must be carefully assessed to exclude potential skin cancers and should be differentiated from those that have a mainly cosmetic consequence. A skin care plan to compensate for failing physiologic function should include good hygiene, pressure care, use of moisturisers and the prevention of occlusive maceration. Conscientious attention to foot care and aggressive treatment of superficial infections may prevent more serious complications. Finally, patients not responding to standard treatment must be referred for specialist management.

\section{See CPD Questionnaire, page 50}

P This article has been peer reviewed

\section{References}

1. Sohal RS, Weindruch R. Oxidative stress, caloric restriction, and aging. Science 1996;273:59-63.

2. Campisi J. Aging, tumor suppression and cancer: high-wire act! Mechanism of Ageing and Development 2005;126:51-8.

3. Krtolica A, Parinello S, Lockett S, Desprez $P$, Campisi J. Senescent fibroblasts promote epithelial cell growth and tumorigenesis. Proc Natl Acad Sci USA 2001;98:12072-7.

4. Fisher GJ, Kang S, Varani J, et al. Mechanisms of photoaging and chronological skin aging. Arch Dermatol 2002; 138:1462-70.

5. Glogan RG. The risk of progression to invasive disease. J Am Acad Dermatol 2000;42:S23

6. Stockfleth E, Meyer T, Benninghof B, et al. A randomized, double-blind vehicle controlled study to assess $5 \%$ imiquimod cream for the treatment of multiple actinic keratosis. Arch Dermatol 2002; 138: 1498-502

7. Gallagher RP, Hill GB, Bajdik CD, et al. Sunlight exposure, pigmentary factors, and risk of nonmelanocytic skin cancer. 1. Basal cell carcinoma. Arch Dermatol 1995;131:157-63.

8. Marks R, Gebauer K, Shumack S, et al Imiquimod 5\% cream in the treatment of superficial basal cell carcinoma: results of a multicenter 6-week dose-response trial. J Am

9. Marcil I, Stern RS. Risk of developing a nonmelanoma skin cancer in patients with a history of nonmelanoma skin cancer. Arch Dermatol 2000,136:1524-30.

10. Grob JJ, Bonerandi JJ. The 'ugly duckling' sign: identification of the common characteristics of reving. Arch 